\title{
Learning to Synchronize: Midfrontal Theta Dynamics during Rule Switching
}

\author{
${ }^{\circledR}$ Pieter Verbeke, ${ }^{\circledR}$ Kate Ergo, ${ }^{\circledR}$ Esther De Loof, and ${ }^{\circledR}$ Tom Verguts \\ Department of Experimental Psychology, Ghent University, Ghent, Belgium B9000
}

In recent years, several hierarchical extensions of well-known learning algorithms have been proposed. For example, when stimulus-action mappings vary across time or context, the brain may learn two or more stimulus-action mappings in separate modules, and additionally (at a hierarchically higher level) learn to appropriately switch between those modules. However, how the brain mechanistically coordinates neural communication to implement such hierarchical learning remains unknown. Therefore, the current study tests a recent computational model that proposed how midfrontal theta oscillations implement such hierarchical learning via the principle of binding by synchrony (Sync model). More specifically, the Sync model uses bursts at theta frequency to flexibly bind appropriate task modules by synchrony. The 64-channel EEG signal was recorded while 27 human subjects (female: 21, male: 6) performed a probabilistic reversal learning task. In line with the Sync model, postfeedback theta power showed a linear relationship with negative prediction errors, but not with positive prediction errors. This relationship was especially pronounced for subjects with better behavioral fit (measured via Akaike information criterion) of the Sync model. Also consistent with Sync model simulations, theta phase-coupling between midfrontal electrodes and temporoparietal electrodes was stronger after negative feedback. Our data suggest that the brain uses theta power and synchronization for flexibly switching between task rule modules, as is useful, for example, when multiple stimulusaction mappings must be retained and used.

Key words: cognitive control; midfrontal theta; neural synchrony; rule switching

Significance Statement

Everyday life requires flexibility in switching between several rules. A key question in understanding this ability is how the brain mechanistically coordinates such switches. The current study tests a recent computational framework (Sync model) that proposed how midfrontal theta oscillations coordinate activity in hierarchically lower task-related areas. In line with predictions of this Sync model, midfrontal theta power was stronger when rule switches were most likely (strong negative prediction error), especially in subjects who obtained a better model fit. Additionally, also theta phase connectivity between midfrontal and task-related areas was increased after negative feedback. Thus, the data provided support for the hypothesis that the brain uses theta power and synchronization for flexibly switching between rules.

\section{Introduction}

Switching between rules is key to function in a complex and rapidly changing environment. For instance, when at the pub with friends, our behavior is likely guided by different social rules than at work. However, when the boss suddenly walks into the pub, this requires to flexibly switch between these two sets of

Received July 19, 2020; revised Nov. 24, 2020; accepted Dec. 2, 2020.

Author contributions: P.V. and T.V. designed research; P.V. and K.E. performed research; P.V., K.E., and E.D.L. analyzed data; P.V. wrote the first draft of the paper; P.V., K.E., E.D.L., and T.V. edited the paper; P.V. and T.V. wrote the paper.

This work was supported by Research Foundation Flanders Grant $1102519 \mathrm{~N}$ to P.V. and Grant $1153418 \mathrm{~N}$ to K.E., E.D.L., and T.V. were supported by Ghent University Research Council Grant BOF17/G0A/004. We thank Clay Holroyd for useful comments on this paper.

The authors declare no competing financial interests.

Correspondence should be addressed to Pieter Verbeke at pjverbek.verbeke@ugent.be.

https://doi.org/10.1523/JNEUROSCI.1874-20.2020

Copyright $\odot 2021$ the authors social rules. Importantly, an empirically valid model that explains how the human brain mechanistically deals with such switches remains lacking.

In experimental settings, this cognitive flexibility in rule switching is typically tested in a reversal learning setup (Izquierdo et al., 2017). Here, agents must learn task rules, each consisting of a collection of stimulus-action mappings. During the task, these rules are regularly reversed. One popular framework to explain performance during reversal learning tasks is the Rescorla-Wagner model (RW) (Widrow and Hoff, 1960; Rescorla and Wagner, 1972). Here, on every trial, obtained reward is used to update the value of active stimulus-action mappings. By learning fast, the agent can flexibly deal with changes in task rules. However, when feedback is probabilistic (e.g., Cools et al., 2002), this approach experiences difficulties. Specifically, a high learning rate will lead agents to "chase the noise" introduced by probabilistic feedback. In contrast, a low learning rate increases robustness against noise 
but decreases flexibility on rule switches. Thus, some researchers have proposed that learning rate should be adaptive (e.g., Behrens et al., 2007; Bai et al., 2014; Silvetti et al., 2018). In this adaptive learning rate (ALR) proposal, agents track rule switches by comparing an estimate of reward probability with received reward. Consistently high prediction errors indicate that the underlying rule has changed, and learning rate should be increased. More fundamentally, however, regardless of learning rate flexibility, both RW and ALR frameworks assume that, on every rule reversal, old information is overwritten. Especially for more complex problems, this is inefficient, as is demonstrated by the problem of catastrophic forgetting in artificial neural networks (French, 1999).

To overcome catastrophic forgetting, separate task rules may be stored (Wilson et al., 2014; Saez et al., 2015). This poses a new problem of keeping track of which task rule is currently relevant. Recent fMRI research focusing on this hierarchical approach toward reversal learning has pointed to midfrontal cortex as the responsible neural structure for keeping track of the current task rule (Wilson et al., 2014). However, how midfrontal cortex mechanistically coordinates neural communication in switching between task rules remains an open question.

This question was recently addressed by a novel computational framework of hierarchical learning (Verbeke and Verguts, 2019). This Sync model retains separate mappings for every task rule, and keeps track of rule reversals by calculating prediction error (e.g., Holroyd and McClure, 2015), thus avoiding catastrophic forgetting. In order to guide neural communication between areas holding the appropriate mappings, the model relies on binding by synchrony (BBS) (Gray and Singer, 1989; Fries, 2005, 2015; Womelsdorf et al., 2007) in theta frequency $(4-8 \mathrm{~Hz})$. Specifically, midfrontal theta oscillations synchronize neuronal activity along task-relevant pathways. Thus, task-relevant neurons can communicate and learn, while stability is achieved in currently irrelevant pathways.

The current study empirically tests this Sync model (Fig. 1A). For this purpose, the model is fitted on data of subjects performing a probabilistic reversal learning paradigm, and empirically compared with alternative models (Rescorla and Wagner, 1972; Bai et al., 2014). Then, Sync model simulations provided several predictions for EEG measured during the task, specifically in theta frequency (model-driven EEG predictions). First, a linear relationship between midfrontal theta power and negative prediction errors was predicted, especially in subjects with good behavioral Sync model fit. Second, a peak of midfrontal theta power was predicted for data locked to rule switches. Third, phase-coupling between midfrontal and posterior electrodes was predicted to be stronger after negative feedback.

\section{Materials and Methods}

\section{Materials}

The experiment was run on a Dell Optiplex 9010 mini-tower running PsychoPy software (Peirce et al., 2019). Electrophysiological data were recorded using a BioSemi ActiveTwo system with $64 \mathrm{Ag} / \mathrm{AgCl}$ electrodes arranged in the standard international 10-20 electrode mapping (Jasper, 1958), with a posterior CMS-DRL electrode pair. Two reference electrodes were positioned at the left and right mastoids. Eye movements were registered with a pair of electrodes above and below the left eye and two additional electrodes at the outer canthi of both eyes. EEG signals were recorded at a sampling rate of $1024 \mathrm{~Hz}$.

Models were fitted using the differential evolution method of the SciPy (version 1.4.1) package in Python (version 3.7.6). Other behavioral analyses were done using R software (R Core Team, 2017). The electrophysiological data were preprocessed in MATLAB R2016b (The MathWorks) using an EEGLAB preprocessing pipeline (Delorme and
Makeig, 2004). Also for simulations of the Sync, model MATLAB R2016b was used.

\section{Code and data accessibility}

All code used to provide the results described in the current paper is provided at https://github.com/CogComNeuroSci/PieterV_public/tree/master/ Reversal_learning. At publication, also the data will be made freely accessible at https://osf.io/wt36f/.

\section{Experimental design}

Both the model (27 simulations) and human subjects $(N=27)$ performed a probabilistic reversal learning task (Fig. 1B). Agents had to learn task rules consisting of two stimulus-action mappings which were regularly reversed during the task. Every trial started with a centrally presented white fixation cross for $2000 \mathrm{~ms}$. Then the stimulus was presented for a period of $100 \mathrm{~ms}$. This stimulus was a centrally presented circular grating with a raised-cosine mask and a size of 7 visual degrees. The grating was either vertically or horizontally oriented. After stimulus presentation, the screen turned blank until response. Responses were given by pressing the ' $\mathrm{f}$ (left) or ' $\mathrm{j}$ ' (right) key on an azerty keyboard. In task Rule 1, the horizontal stimulus mapped to a left response and the vertical stimulus to the right response; this was reversed for task Rule 2. During the task (480 trials), 15 rule switches were introduced. These rule switches occurred at random (uniform distribution from 15 to 45 trials after the previous task switch). After response, probabilistic feedback was presented in the center of the screen. This feedback consisted of ' +10 points' for rewarded trials, ' +0 points' for unrewarded trials or 'Respond faster!' when response times (RTs) were slower than $1000 \mathrm{~ms}$. Subjects had an $80 \%$ probability of receiving reward feedback after correct responses and $20 \%$ after incorrect responses. After feedback, the fixation cross appeared again for another $2000 \mathrm{~ms}$. Crucially, the experiment was divided into two experimental blocks (240 trials each). In one block, the reporting block, the postfeedback fixation cross was presented in green. During this period, subjects were instructed to press the space bar if they thought the task rule had switched. The purpose of this approach was to obtain an indication of when the subject reached his or her own 'Switch threshold,' as happens in the Sync model. This was only done during one block, so critical changes because of this difference in task structure could be checked. The order of the two blocks was counterbalanced across subjects. In between blocks, as well as 3 times within a block, subjects were allowed a short break. This break could only occur if there was no rule switch within 10 trials from the break.

\section{Human testing procedure}

A power analysis considering a linear regression with two predictors (prediction error and reward), a large power of 0.8 and a strong effect size of $R^{2}=0.25$, revealed that 34 subjects needed to be recruited. Seven subjects were removed because of either technical problems with the EEG recording (4) or an inability to give a correct response on $>2 / 3$ of the trials (3), resulting in $N=27\left(N_{\text {male }}=6, N_{\text {female }}=21\right)$. Subjects were told they would receive $€ 25$ for their participation, with a possibility to earn up to $€ 3$ extra reward depending on their performance.

Before starting the task, the subject had to go through two short practice sessions with gratings that were tilted $45^{\circ}$ to the left or to the right relative to a vertical line. In the first practice session, the subject performed 30 trials with only one task rule. Here, the goal was to let the subject get acquainted with the general paradigm and learn a task rule through probabilistic feedback. Subjects were only allowed to continue to the second practice session if they performed above chance level (50\%) and could report the correct task rule to the experimenter. If not, they performed this practice session again. In the second practice session, subjects performed 60 trials of the task with three rule switches and with the postfeedback green fixation cross (as in the reporting block). In this session, subjects pressed the space bar to indicate a task switch and received feedback for each press. The press was considered correct if subjects responded within 10 trials from the actual rule switch. They were allowed to continue to the next task if they were able to perform above chance level and had at least 1 correct indication of a rule switch. 


\section{A}

\section{Switch unit}

Keeps track of, and implements, rule switches

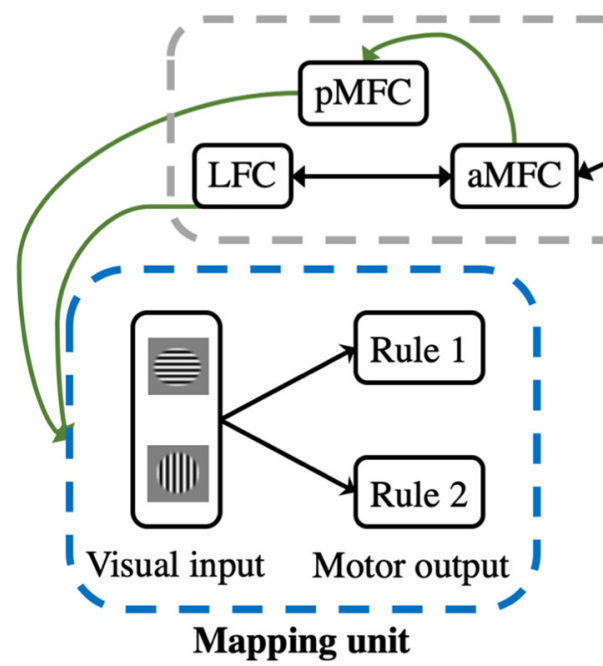

Holds stimulus-action mappings
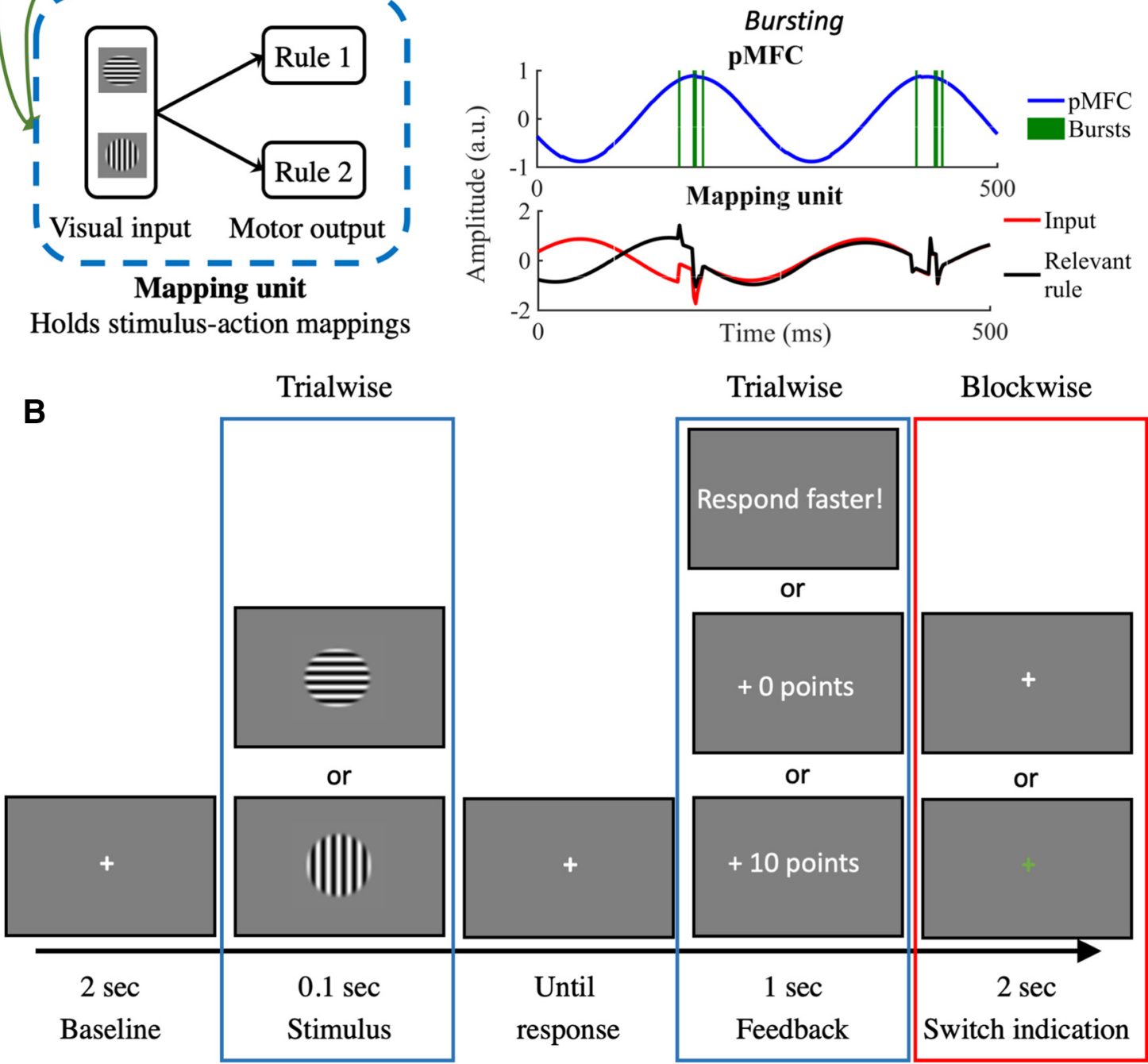

Figure 1. Methods. $\boldsymbol{A}$, The Sync model. Left, Schematic overview of the Sync model. Bottom right, Detailed illustration shows how bursts originating in the pMFC synchronize task-relevant areas in the Mapping unit (for detailed explanation, see Verbeke and Verguts, 2019). $\boldsymbol{B}$, The task. The time course of one trial in the experimental paradigm is shown. Elements highlighted by a blue rectangle, such as the presented stimulus and feedback, are manipulated on a trial-by-trial basis. Elements highlighted by the red rectangle are manipulated blockwise. Here, the fixation cross after feedback was green in one experimental block (half of all trials). In this reporting block, subjects had to press the space bar during this period if they thought the rule had switched.

After successfully performing both practice sessions, subjects performed 480 trials of the actual task.

\section{Statistical analyses}

\section{Behavioral analyses}

To check for differences between the reporting block (green fixation cross) and the nonreporting block (see Experimental task and Fig. 1B), paired $t$ tests were performed for both accuracy and RT, depending on experimental block. In order to deal with the skewed distribution of RT, the natural $\log$ of RT was used for all analyses. Additionally, trials with too late responses (RT $>1000 \mathrm{~ms} ; 2.11 \%$ of all data) were excluded for both behavioral and EEG analyses.
Model analyses

More extensive analyses of behavioral data were done with a modelbased approach. Current work aims to test the Sync model (Verbeke and Verguts, 2019), but two baseline models were fitted as well. In the following section, we first provide a detailed overview of the Sync model, followed by a description of all three models that were fitted on behavioral data. Then, we describe how model fit was evaluated.

The Sync model. An overview of model architecture is provided in Figure $1 A$. The Sync model consists of two units: the Mapping and Switch unit. The Mapping unit contains a classic network with 2 layers (visual input and motor output). Here, weights are adapted with the RW algorithm (Widrow and Hoff, 1960). In the Sync model, 4 nodes (2 for each response option) at the motor output layer are divided in 2 rule 
modules, one for each task rule. Hence, as in Wilson et al. (2014), the Mapping unit holds separate stimulus-action mappings for each task rule. In addition, a Switch unit forms a hierarchically higher network modeled after primate PFC. This Switch unit keeps track of switches in task rule. Specifically, the Switch unit consists of the lateral frontal cortex (LFC), posterior medial frontal cortex (pMFC), and anterior midfrontal cortex (aMFC). Here, the LFC holds pointers (e.g., Cohen et al., 1990; Botvinick et al., 2001) that indicate which rule should be synchronized in the Mapping unit. Since BBS implements gating, allowing efficient communication between synchronized nodes and blocking communication between nonsynchronized nodes (Fries, 2005, 2015), the agents' behavior will be guided by the synchronized rule. This synchronization process is then executed by the binding by random bursts principle (Zhou et al., 2005; Springer and Paulsson, 2006; Verguts, 2017). In the Sync model, a theta-frequency-paced signal produced in the pMFC is responsible for sending these bursts (for details, see Verguts, 2017; Verbeke and Verguts, 2019). The aMFC contains a neural network (for simplicity not shown in Fig. $1 A$ ) that is adapted from previous work (Silvetti et al., 2011). Here, again RW learning is used but on a hierarchically higher level. More specifically, the aMFC learns an expected reward $(V)$ for the currently used rule module (see Eq. 6). This expected reward is compared with an external reward signal (Rew; Reward in Fig. 1A) to compute prediction errors. The negative prediction error signal is propagated to both the Accumulator neuron (within the aMFC neural network) and to pMFC. A single negative prediction error increases (via bursting) the power of the theta signal in pMFC (bursting connection in Fig. 1A) (for details, see Verbeke and Verguts, 2019). Instead, the Accumulator neuron evaluates the prediction error signal on a slower time scale (see also Holroyd and McClure, 2015), and thus requires multiple prediction errors before activation in the Accumulator neuron reaches its Switch threshold (see Eq. 5). When this happens, aMFC signals the need for a switch to the LFC. Correspondingly, the LFC will change the signal to the Mapping unit, and synchronize another rule module. In sum, bursts received by the Mapping unit are the result of a cooperation between LFC and pMFC. The pMFC determines the intensity of theta bursts, whereas the LFC determines which task rule in the Mapping unit is susceptible to the bursts (for further details, see Verbeke and Verguts, 2019).

All nodes in the visual input and motor output layer of the Mapping unit as well as the pMFC are oscillatory nodes. In line with previous work (Verguts, 2017), oscillatory nodes consist of neuronal triplets. The neural triplet contains one excitatory-inhibitory pair of phase code neurons $(\mathrm{E}, \mathrm{I})$ and a rate code neuron. Here, excitatory neurons are updated by the following:

$$
\Delta E_{i}(t)=C I_{i}(t)-D \times J\left(r>r_{\min }\right) \times E_{i}(t)+B_{i}(t)
$$

where $\Delta E(t)=E(\mathrm{t}+\Delta t)-E(t)$; and inhibitory neurons are updated by the following:

$$
\Delta I_{i}(t)=-C E_{i}(t)-D \times \mathrm{J}\left(r>r_{\min }\right) \times I_{i}(t)
$$

Here, phase code neurons will oscillate at a frequency of $C / 2 \pi$. In the pMFC, which executes top-down control by sending bursts, activity oscillates at theta $(6 \mathrm{~Hz})$ frequency, in line with suggestions of previous empirical work (Womelsdorf et al., 2010; Cavanagh and Frank, 2014). Different from our previous modeling work, theta frequency was used in the Mapping unit (see Discussion) as well. Because bursts $(B(\mathrm{t}))$ lead to a significant increase of power, a radius parameter $\left(r_{\text {min }}\right)$ is implemented to attract power $(r)$ back to baseline after a burst. Since continuously high pMFC power is computationally suboptimal and empirically implausible (Holroyd, 2016), power in the pMFC was attracted toward a smaller radius $\left(r_{\min }=0.50\right)$ than in the Mapping unit $\left(r_{\min }=1\right)$. How fast oscillations decay to baseline is determined by a damping parameter $(D)$, which was set to $D=0.30$ in the Mapping unit. Since the pMFC not only receives bursts but also sends them, a slower decay $D=0.01$ was implemented here to allow a sufficient activity window $(\sim 500 \mathrm{~ms} / 3$ theta cycles) for bursts to be sent. In order to reduce model complexity, no oscillations were used in the LFC and aMFC. For a full description of model dynamics, see Verbeke and Verguts (2019).
Thus, in the Sync model, on every trial, multiple time steps were simulated in which oscillations occurred. Here, motor nodes accumulate activation over time. The motor node with the maximal accumulated activation over time was considered as the model response. Values of stimulus action pairs $(Q)$ in each rule module $(R)$ are updated by the following:

$$
Q(s, a)_{j+1}=Q(s, a)_{j}+\alpha *\left(\operatorname{Rew}-Q(s, a)_{j}\right)
$$

in which $\alpha$ is the Mapping learning rate and Rew is the reward received by the agent.

As described above, the Sync model has an additional Switch unit which adds a hierarchical learning algorithm on top of the RW (fixed learning rate) algorithm in the Mapping unit. This Switch unit evaluates whether there was a rule switch. More specifically, it learns a value $(V)$ for every rule module $(R)$ by the following:

$$
V(R)_{j+1}=V(R)_{j}+\alpha_{h i g h} *\left(R e w-V(R)_{j}\right)
$$

in which $\alpha_{\text {high }}$ is the hierarchically higher Switch learning rate. The difference between the expected value $V(R)$ in Equation 6 and the obtained Rew (i.e., the prediction error) is accumulated in the Accumulator neuron $(A)$ via the following:

$$
A_{j+1}=\gamma * A_{j}+(1-\gamma) * f\left(\operatorname{Rew}-V(R)_{j}\right)
$$

Since switches are only required when negative feedback occurs, the Accumulator neuron was selective for negative prediction errors. Specifically, $f($ Rew $-V(R))=-($ Rew $-V(R))$ when the prediction error is negative and $f($ Rew $-V(R))=0$ when the prediction error is positive. Here, $\gamma$ is the Cumulation parameter, which determines how strongly the Accumulation neuron is affected by a single prediction error. While a low Cumulation parameter causes the agent to strongly weigh single prediction error and therefore regularly switch between rule modules, a high Cumulation parameter implements a more conservative approach. When the Accumulator neuron reaches a Switch threshold of 0.5 , the model will switch to another rule module $(R)$ in the Mapping unit.

Behavioral data fitting. For behavioral data fitting only, the full Sync model was simplified by introducing a hard gating process between task rules instead of BBS and a softmax response selection mechanism described by the following:

$$
p(a=i)=\frac{e^{Q(s, i) / \tau}}{\sum_{a=1}^{2} e^{Q(s, a) / \tau}}
$$

in which $Q(s, a)$ is the value of a given stimulus-action pair $(s, a)$ and $\tau$ is the temperature parameter which determines how strongly the agent explores different actions (1). This allowed to skip the loop of 1500 time steps every trial, which was needed to simulate oscillations (see Eqs. 1,2). We refer to this model as the behavioral Sync (bSync) model.

On top of the bSync model, two other models were fitted as well. The RW and ALR model are both restricted to only the Mapping unit (with one rule module). Both models use a response selection mechanism as described by Equation 6 and learn stimulus-action pairs by Equation 3 . Importantly, the RW model (Rescorla and Wagner, 1972) had a constant learning rate, whereas the ALR model (Bai et al., 2014) was implemented with an adaptable learning rate. Here, the Mapping learning rate is updated on every trial by the following:

$$
\alpha_{j+1}=\eta *\left|\operatorname{Rew}-Q(s, a)_{j}\right|+(1-\eta) * \alpha_{j}
$$

in which $\eta$ determines how strongly the learning rate is influenced by the current difference between Rew and $Q$ (lower-level prediction error). 
Model evaluation. For each subject, the goodness of fit of these three models on the behavioral data was compared by using three measures. The log-likelihood $(L L)$ is as follows:

$$
L L=\sum_{j=1}^{J} a_{j} * \ln \left(p\left(a_{j}=1\right)\right)+\left(1-a_{j}\right) * \ln \left(p\left(a_{j}=0\right)\right)
$$

in which $p(a)$ is the probability of the given action (see Eq. 6) and $J$ indicates the number of trials. The Akaike information criterion (AIC) uses this $L L$ but includes a penalty for the number of parameters $(k ; k=2$ for $\mathrm{RW}, k=3$ for ALR, and $k=4$ for bSync) that were used in the model as follows:

$$
A I C=2 * k-2 * L L
$$

From this AIC, AIC weights ( $w A I C)$ can be derived, which allow to make a relative comparison between the model fit of the three different models. These wAIC values are computed as follows:

$$
w A I C=\frac{e^{-\frac{1}{2} \Delta A I C_{m}}}{\sum_{m=1}^{M} e^{-\frac{1}{2} \Delta A I C_{m}}}
$$

in which $M$ is the number of models that are compared (mean $=3)$ and

$$
\Delta A I C_{m}=A I C_{m}-\min (A I C)
$$

Here, min(AIC) is the lowest AIC value out of the three models for that subject. Thus, Equation 10 results in a wAIC value for each model. The sum of all three wAIC values is 1 and models with higher wAIC values provide a better fit to the data.

Simulations. In order to provide hypotheses for EEG data, 27 simulations of the full Sync model were performed. For all simulations, the same parameter values were used. These parameter values were sampled from the distribution of best fitting parameter values of the bSync model so that overall accuracy of model simulations $($ mean $=78.00 \%, \mathrm{SD}=1.30)$ closely resembled accuracy of subjects (mean $=76.80 \%, \mathrm{SD}=4.91$ ). This resulted in a Mapping learning rate $(\alpha)$ of 0.8 , a Switch learning rate $\left(\alpha_{\text {high }}\right)$ of 0.1 , and a Cumulation parameter $(\gamma)$ of 0.3. The full Sync model did not use a Temperature $(\tau)$ parameter; instead, the synchronization procedure introduces noise, which also introduces some randomness in behavior. The Switch threshold was always fixed to 0.5. Trials were simulated as a fixed period of $500 \mathrm{~ms}$ in which the visual layer received stimulation. After this period, the response node with the highest maximum activation was registered as the response of the model. Thereafter, $1500 \mathrm{~ms}$ of intertrial interval was simulated to provide a postfeedback period that could be analyzed in the same way as the empirical data. All other aspects of the task, such as the frequency and timing of rule switches, were the same for the model as for the human subjects.

Power analyses. Time-frequency decomposition was performed on the excitatory neuron (see Eq. 1) within the neuronal triplet of the model's pMFC node in the model. Complex Morlet wavelets were used for frequencies between 2 and $48 \mathrm{~Hz}$ defined in 25 logarithmically spaced steps. For each frequency, between 3 and 8 cycles were used, also defined in 25 logarithmically spaced steps. Power was extracted as the squared absolute value of the time-frequency decomposed signal. In order to locate activity that was specific to feedback processing, the difference between power in trials with negative feedback and trials with positive feedback was computed. For simplicity, we selected the $2.5 \%$ most positive values as a cluster of interest. This cluster contained one group of data points in theta frequency and $\sim 250-500 \mathrm{~ms}$ after feedback (for timing details, see Verbeke and Verguts, 2019). On every trial, the mean power in this cluster was computed and entered in the consecutive analyses. Since a negative prediction error in the model increases activity of the pMFC, we performed a linear regression of cluster power with prediction error as the independent variable. To test our first hypothesis, that this relationship was specific to negative prediction error, a second regression model was used that also included the interaction between prediction error and reward. The second hypothesis states that, because negative prediction errors are strongest at the moment of a rule switch, a peak of postfeedback theta power should be found when data are locked to rule switches. To investigate this, we extracted power from the model cluster in trials within a 31-trial window around the rule switch $(-15$ to 15). The time course (one data point for each of the 31 trials) that resulted after averaging over all (15) rule switches and all (27) simulations was then used as a regressor in a linear regression with data from the empirical clusters.

Phase analyses. Our third hypothesis stated that phase-coupling between pMFC and model nodes in the Mapping unit was stronger after negative feedback. Specifically, theta power in the model pMFC increases after negative prediction errors. When there is sufficient power in the pMFC, it will increase synchronization in the Mapping unit (posterior/ lateral task-related regions, e.g., premotor or visual areas). For this purpose, the pMFC uses binding by random bursts (Verguts, 2017). Here, the pMFC will send bursts to the Mapping unit at specific phases. Thereby it will shift the phase of neurons in the Mapping unit (for details, see Verbeke and Verguts, 2019). This leads to phase shifts in these lower premotor or visual task-related areas, and a short period of phase-alignment between these task-related areas in the Mapping unit and pMFC. Phase was extracted in all model nodes by taking the angle of the Hilbert transform of the raw signal. For simplicity, the model was implemented without interareal delays. Furthermore, in contrast to analyses on the empirical EEG data (see Eq. 12), control for volume conduction was not needed, so the regular phase locking value (PLV) (Lachaux et al., 1999) was computed between the model pMFC and the nodes in the motor layer of the Mapping unit. This PLV was then averaged over all four motor nodes and the time period included in the power cluster ( $\sim 250-500 \mathrm{~ms}$ after feedback).

\section{EEG analyses}

Preprocessing. The data were rereferenced offline to the average of the mastoid electrodes. Breaks or other offline periods were manually removed. Particularly noisy electrodes were interpolated between neighboring electrodes on all time steps. For 3 subjects, one electrode was interpolated; for another 3 subjects, we had to interpolate two electrodes; because of a bridge, 1 subject needed interpolation for five posterior electrodes. Additionally, activity was bandpass filtered between 1 and $48 \mathrm{~Hz}$ to remove slow drifts and line noise of $50 \mathrm{~Hz}$. Eyeblinks and other motor-related noise components were removed through EEGLAB independent component analysis. After independent component analysis removal, the data were epoched, once locked to feedback onset, and once to stimulus onset. The epochs based on stimulus onset were used to extract baseline activation, which was -1500 to $-500 \mathrm{~ms}$ relative to stimulus onset. This baseline activity was subtracted from all epochs. After epoching, on average, $7.5 \%$ of epochs were removed by applying an amplitude threshold of $-500-500 \mathrm{mV}$ and an improbability test with 6 SDs for single electrodes and 2 SDs for all electrodes, as described in Makoto's preprocessing pipeline (Makoto, 2018). Before time-frequency analyses, data were also downsampled to $512 \mathrm{~Hz}$.

Time-frequency decomposition. Time-frequency decomposition was based on code from Cohen (2014). Similar to model analyses, complex Morlet wavelets were used for frequencies between 2 and $48 \mathrm{~Hz}$ defined in 25 logarithmically spaced steps. For each frequency, between 3 and 8 cycles were used, also defined in 25 logarithmically spaced steps.

Power computation. A baseline correction was applied by dividing the power estimates for each subject, electrode, and frequency by the average baseline activity $(-1500 \mathrm{~ms}$ to $-500 \mathrm{~ms}$ from stimulus onset) across all 480 trials. Finally, the baseline-corrected data underwent a decibel conversion. Before final analyses, also trials with late responses were removed from the data.

Power cluster analyses. Similar to model analyses, we were interested in activity selective for feedback. Hence, a contrast between $z$-scored power in trials with negative feedback and trials with positive feedback was computed. On these values, a nonparametric clustering procedure 
was applied (Maris and Oostenveld, 2007). The distribution of statistics was computed. On each side of the distribution (two-sided test), the $1 \%$ most extreme values were entered into the clustering analysis. From these, we clustered adjacent neighbors in the channel, frequency, and time domains. To calculate our cluster-level statistic, we multiplied the number of items (i.e., channel, frequency, time points) in the cluster with the largest statistic of that cluster (see also Maris and Oostenveld, 2007). A significance threshold of $5 \%$ was imposed on the subsequent nonparametric permutation test with 1000 iterations. Clusters that survived this permutation test were taken into the consecutive analyses. As an exploratory analysis, we aimed to link individual differences in behavioral model fit to EEG data; for that purpose, we extracted the mean cluster statistic for each subject, and ran a Spearman rank correlation of these statistics with wAIC of the bSync model obtained in the behavioral model fitting procedure.

Midfrontal theta power and prediction error. The Sync model uniquely yields specific EEG predictions, to which we now turn. To test the first model-driven EEG hypothesis of a relation between theta power and prediction errors, we first extracted a measure of prediction error for every subject on every trial by simulating the bSync model. Importantly, this prediction error was extracted from the learning process on the hierarchically higher level in the Switch unit (Eq. 4), not the lower-level learning process in the Mapping unit (Eq. 3). This measure of prediction error was then used in a trial-by-trial linear mixed effects model as a predictor for the $z$-scored power of every cluster (averaged across all time, electrode, and frequency points in the cluster), that survived the feedback-locked analysis described above. Here, a random intercept for every subject was included and a fixed slope (i.e., the prediction error). Because the Sync model predicted different relationships for positive prediction errors and negative prediction errors, also the interaction between prediction errors and reward was tested. Additionally, to explore whether the individual differences in wAIC influenced the interaction between prediction errors and reward, also a three-way interaction between prediction error, reward, and wAIC was tested. For these purposes, three regression models were fitted: One in which only prediction error was included as regressor, one in which both prediction error and the interaction between prediction error and reward were included as regressors, and finally a third model in which the main effect, the two-way interaction, and an extra three-way interaction between prediction error, reward, and wAIC were included as regressors. These regression models were then compared via ANOVA.

Rule switch locking. A second model-driven EEG hypothesis considers theta power locked to the moment of a rule switch. For this analysis, EEG data of 31 trials around the rule switch ( -15 to 15 trials, including the rule switch trial itself) were extracted. On these trials, the mean power within each cluster selective for feedback was computed. This data were then again averaged over all trials at a specific distance $(-15$ to 15) from switch, giving us a time course of mean cluster-power from -15 trials before rule switch to 15 trials after rule switch for every subject. On each time point, a $99.84 \%$ CI was computed based on a Bonferroni correction for multiple comparisons (100 - (5/31)). This CI was compared with a baseline power. Baseline power was computed based on the mean power in this cluster, averaged over all trials that were $>15$ trials removed from the rule switch.

As the rule switch trial, we considered in separate analyses both the actual rule switch and the subjective indication of a rule switch. Hence, power close to a rule switch was compared with the mean power of trials that were far from the rule switch. When the CI did not include the baseline value, power on this trial was considered as significantly deviating from baseline. Additionally, we aimed to investigate the similarity between the data pattern predicted by the model and the empirical data. For this purpose, data from the bSync model simulations (see above for details) were used as a linear regressor for the empirical data. Also, for this hypothesis, an extra analysis was performed to investigate whether wAIC had an influence on the observed effect. Here, we extracted subject data on trials of which cluster power significantly deviated from baseline and used this data as a dependent variable in a linear regression with wAIC.

Midfrontal-posterior phase-coupling analyses. For the third modeldriven EEG hypothesis, we considered all midline electrodes (10) as seed and other electrodes (54) as receiver in the phase connectivity analyses. Because we were interested in phase-locking related to rule modules conveying the correct response, all data were lateralized with respect to the correct response. All data ipsilateral to the correct response were brought to the left electrodes; all contralateral data were brought to the right electrodes. The iPLV (Bruña et al., 2018) was computed between all midline electrodes and all lateral electrodes for every time point in the feedback-locked data. This iPLV measure was computed by the following equation:

$$
i P L V=\left|\frac{1}{n} \sum_{t=1}^{n} \operatorname{Im}\left(e^{-i\left(\Delta \varphi_{t}\right)}\right)\right|
$$

which computes the average phase angle $(\varphi)$ difference over trials $(t)$. By only looking at the imaginary (Im) part of this phase angle difference, phase differences of zero are eliminated. Hence, volume conduction effects are excluded because such volume conduction effects are represented in zero-phase differences (Nolte et al., 2004; Bruña et al., 2018). Again, a nonparametric cluster algorithm was performed on the contrast between iPLV for trials with negative versus positive feedback (the fact that our effect of interest compares negative vs positive feedback also safeguards against possible volume conduction effects). For this analysis, only data of one midline electrode were used. More specifically, we checked on which of the 10 midline electrodes the mean contrast in the theta frequency $(4-8 \mathrm{~Hz})$ reached a maximum. This was in the FCz electrode; hence, only iPLV between $\mathrm{FCz}$ and all lateral electrodes were entered in the clustering algorithm. As for power, an exploratory analysis was performed in which we extracted the mean cluster statistic for each subject, and ran a Spearman rank correlation of these statistics with wAIC of the bSync model obtained in the model fitting procedure.

\section{Results}

\section{Behavioral data}

Overall, participants had a mean accuracy of $76.80 \%(\mathrm{SD}=4.92 \%)$ and a mean RT of $544 \mathrm{~ms}(\mathrm{SD}=71.31 \mathrm{~ms})$. A paired $t$ test confirmed that there were no significant differences between the experiment block in which subjects had to indicate when a task switch happened or when they did not have to indicate this (for details, see Materials and Methods), neither in accuracy $\left(t_{(26)}=0.029, R^{2}<\right.$ $0.001, p=0.977)$ nor in RT $\left(t_{(26)}=-1.290, R^{2}=0.062, p=0.208\right)$.

\section{Model analyses}

The distribution of all fitted parameter values for each model is given in Figure 2A. Goodness-of-fit measures are summarized in Table 1. Here, LL was highest (best) for the bSync model, lowest for the ALR model, with the RW model in between. When a penalty for model complexity was applied (AIC, wAIC), the RW and bSync models performed approximately equal. Importantly, wAIC results indicated differences across individuals. As illustrated in Figure $2 B$, subjects could be roughly divided into three groups based on the wAIC. In one group ( 8 subjects), the wAIC were significantly smaller (worse) for the bSync model (mean $=0.12$, $\mathrm{SD}=0.026)$ than for the RW model (mean $=0.78, \mathrm{SD}=0.027)$. A second group (7 subjects) showed wAIC values that were approximately equally strong for the bSync (mean $=0.44, \mathrm{SD}=0.036)$ as for the RW model (mean $=0.50, \mathrm{SD}=0.032)$. In a third group $(12$ subjects), the bSync model showed wAIC that were significantly higher for the bSync model (mean $=0.64, \mathrm{SD}=0.027$ ) than for the $\mathrm{RW}$ model $($ mean $=0.32, \mathrm{SD}=0.026)$.

Three parameters of the bSync model showed a significant correlation with wAIC (Fig. 2C). These parameters were the Switch learning rate $(\rho=-0.761, p<0.001)$, the cumulation parameter $(\rho=-0.708, p<0.001)$, and the temperature parameter ( $\rho=-0.497, p=0.008)$. There was no significant correlation with 
A

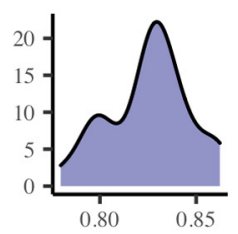

Mapping Learnrate
RW model

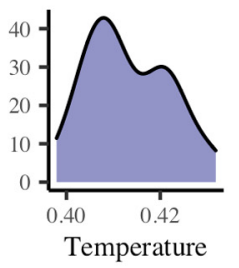

ALR model

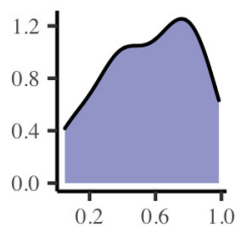

Mapping Learnrate

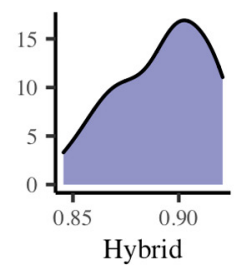

C
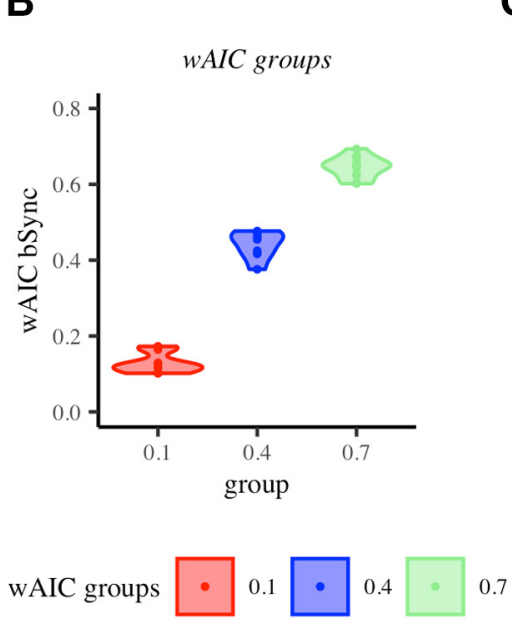

D

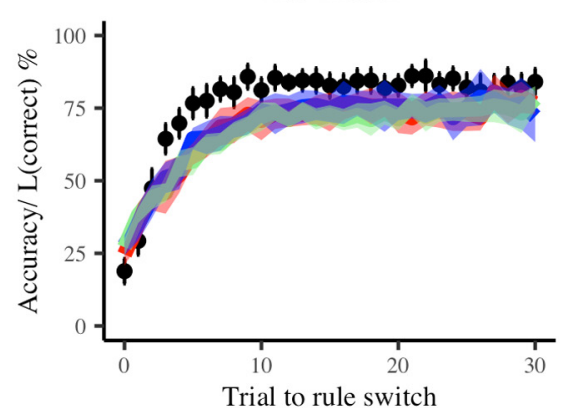

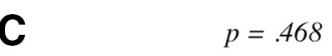

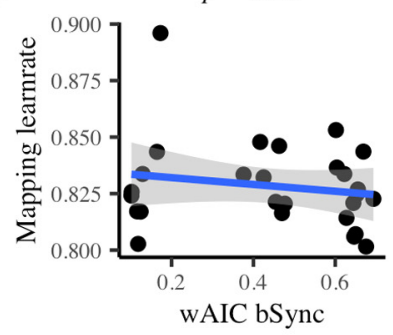

$p<.001$

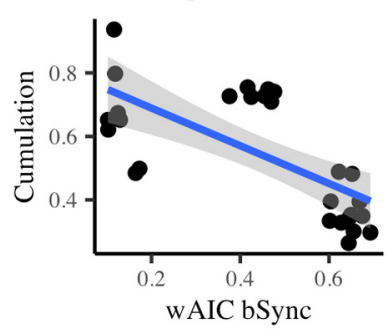

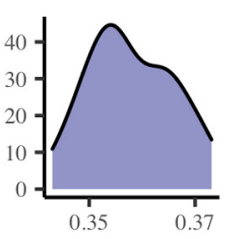

Temperature
bSync model

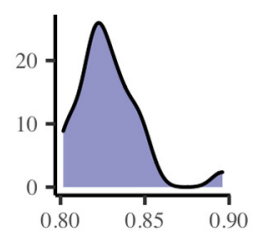

Mapping Learnrate

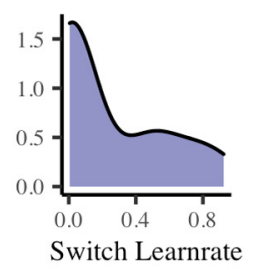

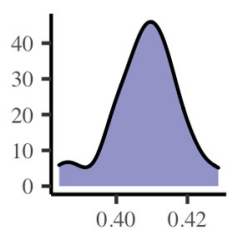

Temperature

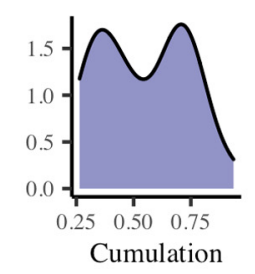

$p=.008$

$p<.001$
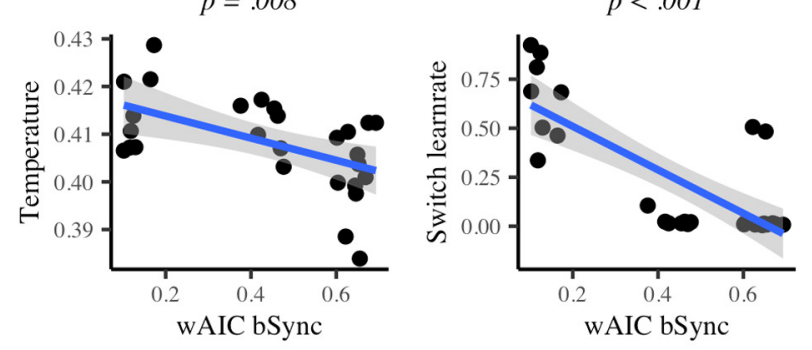

$p=.007$

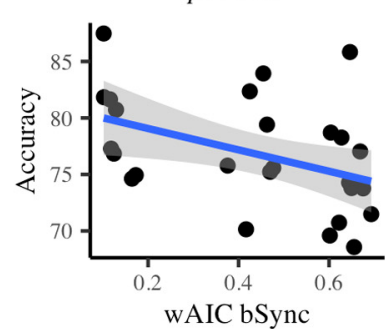

ALR Model
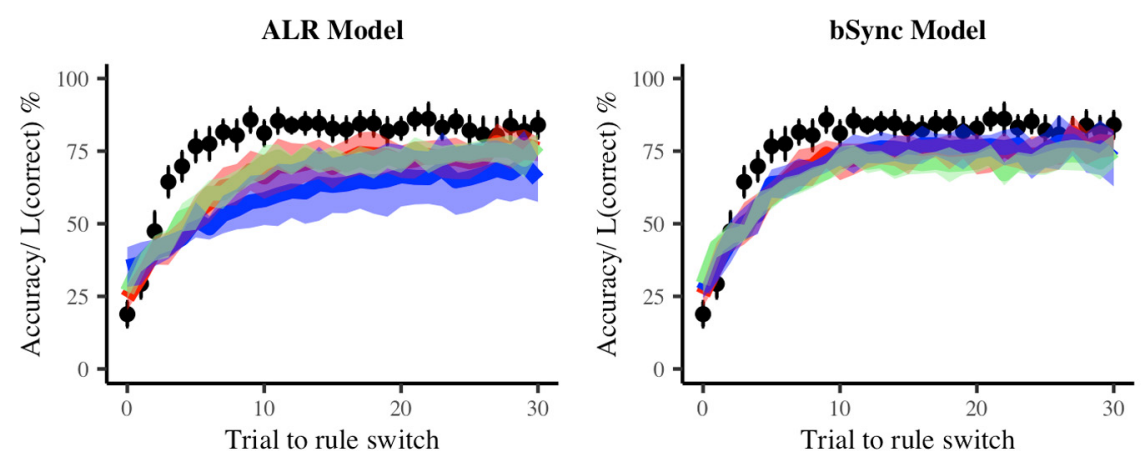

Figure 2. Model comparison. A, Parameter distributions. Distributions of fitted parameter values are shown for each model. $\boldsymbol{B}$, wAIC groups. This figure illustrates how wAIC values can be roughly divided in three groups (colors). C, Correlation plots. Correlations are shown between wAIC of the bSync model and all parameters of the bSync model. Bottom middle, Correlation between wAIC and task accuracy. D, Learning curve fit. Black dots represent the mean accuracy data over all subjects. Error bars indicate the $95 \%$ Cls. Colored lines indicate the mean likelihood of the correct response for each wAIC group in $\boldsymbol{B}$. Shades represent the $95 \% \mathrm{Cl}$.

Table 1. Goodness-of-fit measures ${ }^{\mathrm{a}}$

\begin{tabular}{lllllll}
\hline Model & Mean LL & SD LL & Mean AIC & SD AIC & Mean wAIC & SD wAIC \\
\hline RW & -208.08 & 0.07 & 420.16 & 0.13 & 0.51 & 0.20 \\
ALR & -209.31 & 0.05 & 424.63 & 0.10 & 0.05 & 0.02 \\
bSync & -206.35 & 1.11 & 420.70 & 2.22 & 0.44 & 0.22 \\
\hline
\end{tabular}

${ }^{a}$ Results of LL, AIC, and wAIC computations over subjects are shown for each of three models. For LL and wAIC, high values indicate a better fit, whereas for AIC a low value indicates a good fit. the Mapping learning rate ( $\rho=-0.145, p=0.468$ ). Additionally, a correlation test between accuracy and wAIC revealed that the bSync model fitted significantly better for subjects with a lower accuracy $(\rho=-0.510, p=0.007)$. Also, correlations between wAIC values and parameters of the other two models were tested, but none of these correlations reached significance.

We next estimated a learning curve for each model and each wAIC group (Fig. 2D). This learning curve represents the estimated likelihood of the correct response averaged over all rule 


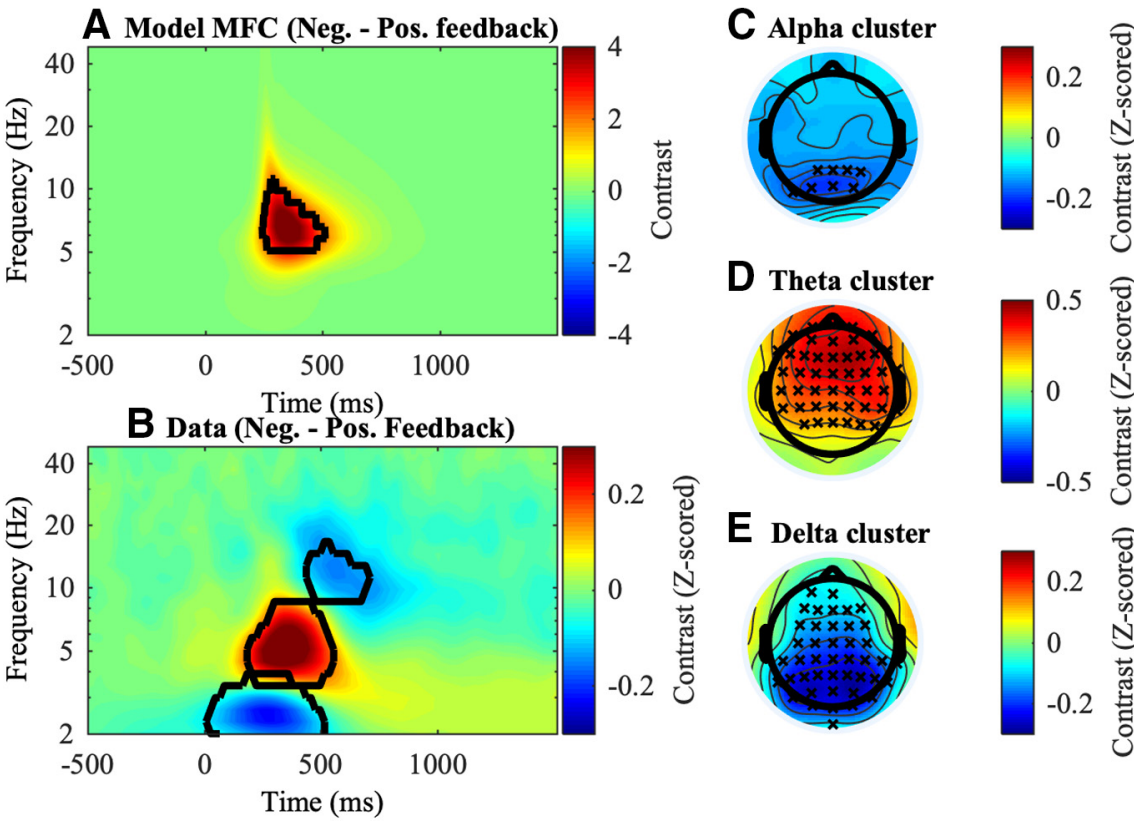

Figure 3. Power results. $\boldsymbol{A}, \boldsymbol{B}$, Time-frequency plots of contrast (Negative - Positive feedback). Black contour line indicates significant clusters. $\boldsymbol{A}$, Contrast of power in the model pMFC. $\boldsymbol{B}$, Contrast for $\boldsymbol{z}$-scored power in the human data, averaged over all 64 electrodes. $\mathbf{C}-\boldsymbol{E}$, Topographical plots of clusters found in the human data. Crosses represent channels where the contrast reached significance.

switches and all subjects within a group. Differences in learning curve between the three groups are very subtle: We conclude that an average measure like switch-locked learning curve does not suffice to empirically distinguish between the three models.

As described in Equation 5, the bSync model only uses negative prediction errors to evaluate rule switches. Nevertheless, it might be argued that also positive prediction errors determine rule switching. To test this, also an alternative version of the bSync model (bSync-linear) was fitted. Here, $f(\operatorname{Rew}-V(R))=$ $-($ Rew $-V(R))$ for all trials. Hence, switch evidence increased for negative prediction errors and decreased for positive prediction errors. Here, we observed a clear advantage in terms of AIC for the original bSync model (mean $=420.70, \mathrm{SD}=2.22$ ) compared with the alternative bSync-linear model $($ mean $=481.12, \mathrm{SD}=$ 75.28). Hence, only the original bSync model was used for the consecutive analyses.

In sum, we found that, for the bSync model, participants' behavior is best explained by the model version that is biased toward negative prediction errors to evaluate rule switches. When comparing this bSync model with the RW and ALR models, three groups of participants could be distinguished. Moreover, the individual measures of model fit correlated significantly with accuracy and several parameters of the bSync model.

\section{EEG and model data}

\section{Power cluster analyses}

Cluster analysis on postfeedback power revealed three significant clusters that were selective for feedback processing (Fig. 3). All three clusters appeared between 0 and $750 \mathrm{~ms}$ from feedback onset. As was predicted by the Sync model (Fig. $3 A$ ), one of these clusters was in the theta frequency range $(\sim 4-8 \mathrm{~Hz})$ and located on midfrontal electrodes (Fig. $3 B, D$ ). This theta cluster showed more power for negative than for positive feedback. Additionally, we found two clusters located on the posterior channels. One of these clusters was in the $\delta$ frequency
$(<4 \mathrm{~Hz}$; Fig. $3 B, E)$, the other cluster was located in the $\alpha$-frequency range $(\sim 8$ $15 \mathrm{~Hz}$; Fig. $3 B, C)$. Both the $\delta$ and $\alpha$ cluster showed less power for negative feedback than for positive feedback. No correlation between the power contrast of a cluster and subjects' wAIC for the bSync reached significance.

Midfrontal theta power and prediction error We next consider the first of three modeldriven EEG hypotheses. We first perform statistical analysis on the Sync-model simulated data (Fig. 4A). Theta power in the Sync model data was best predicted by the regression model that included an interaction between reward and prediction error $\left(F_{(1,11980)}=22,133, R^{2}=0.985\right.$, $p<0.001)$. Hence, there was a significant main effect of prediction error $\left(F_{(1,11980)}=\right.$ 742,962, $R^{2}=0.956, p<0.001, \beta=$ $-4.99)$ and a significant interaction of prediction error and reward $\left(F_{(1,11980)}=\right.$ $\left.22,133, R^{2}=0.985, p<0.001, \beta=4.48\right)$. Thus, as predicted, the model cluster showed a negative linear relationship with negative prediction error, and no linear relationship with positive prediction error (Fig. 4A).

In order to test this prediction in the empirical theta cluster (cluster reported in the previous section), prediction errors were estimated by simulating the bSync model (Fig. 5A). Importantly, these prediction error estimates were extracted from learning in the Switch unit (see Eq. 4) and not from the learning of stimulus-action pairs in the Mapping unit (Eq. 3). For theta power, the regression model, including the interaction between prediction error and reward, fitted significantly better than the regression model with only prediction error as regressor $\left(\chi^{2}(1, N=\right.$ $\left.27)=110, R^{2}=0.096, p<0.001\right)$. Additionally, the regression model, including the three-way interaction between prediction error, reward, and wAIC, fitted significantly better than the regression model with only the two-way interaction $\left(\chi^{2}(2, N=\right.$ $\left.27)=20.74, R^{2}=0.021, p<0.001\right)$. Here, all effects reached significance. Hence, there was a main effect of prediction error $\left(\chi^{2}(1, N=27)=1299, R^{2}=0.329, p<0.001, \beta=-0.79\right)$ and an interaction of prediction error with reward $\left(\chi^{2}(1, N=27)=\right.$ $\left.110, R^{2}=0.096, p<0.001, \beta=0.65\right)$. Additionally, there was a significant interaction between prediction error, reward, and wAIC $\left(\chi^{2}(2, N=27)=20.90, R^{2}=0.042, p<0.001\right)$. As can be observed in Figure $4 B$, these results indicated a significant negative linear relationship between power and negative prediction error, which was stronger for subjects with a high wAIC (i.e., better behavioral fit of the Sync model); and an absence of linear relationship between power and positive prediction error which did not differ significantly for wAIC (Fig. 4B). Interestingly, the three-way interaction was significant in the unrewarded (negative prediction error) trials $\left(R^{2}=0.029, \beta=-0.89, p<0.001\right)$ but did not reach significance in the rewarded (positive prediction error) trials $\left(R^{2}=0.014, \beta=0.44, p=0.077\right)$.

For exploratory purposes, we investigated the same regression models in the $\delta$ and $\alpha$ clusters. In the $\delta$ cluster, the difference in regression model fit between the regression models without and with the prediction error-reward interaction term did not reach significance $\left(\chi^{2}(1, N=27)=3.49, R^{2}=0.017, p=0.062\right)$. 
A

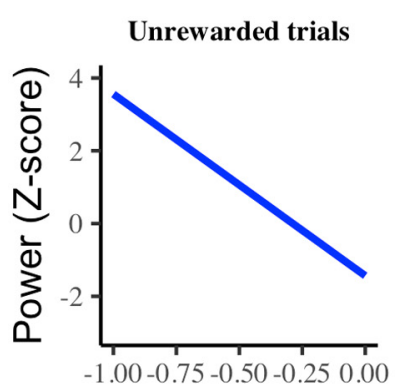

Model cluster

\section{Rewarded trials}

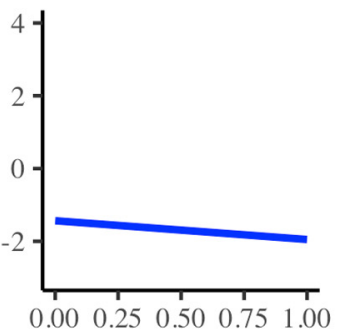

B

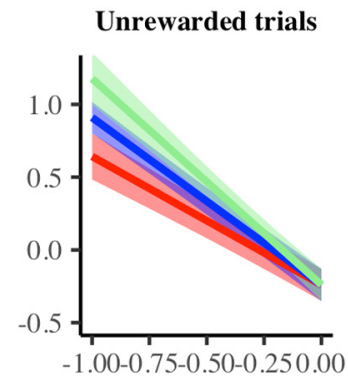

Theta cluster

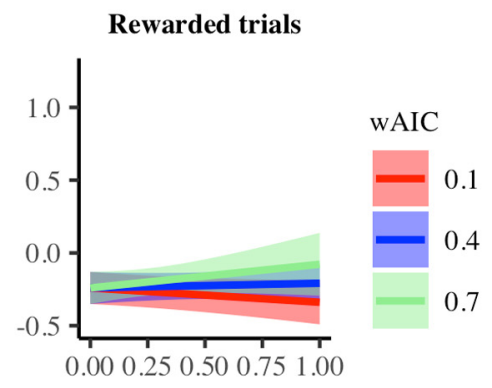

C

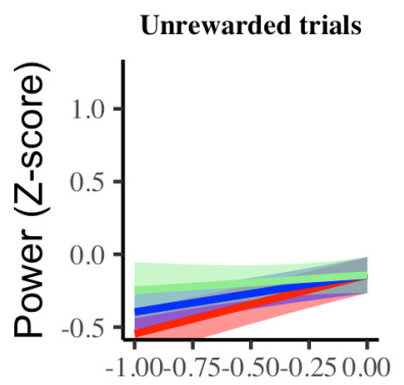

PE
Delta cluster

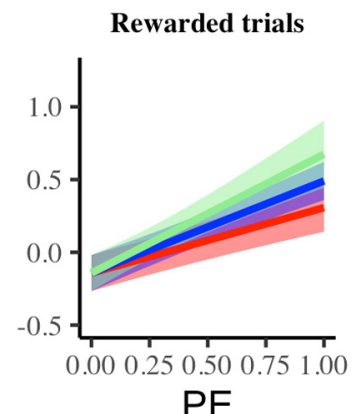

D

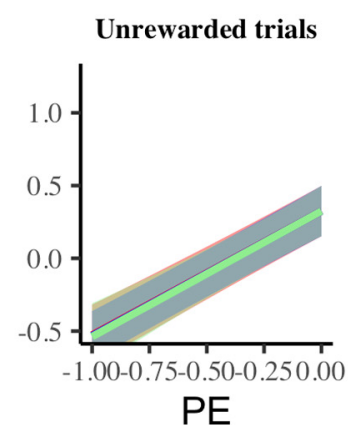

Alpha cluster

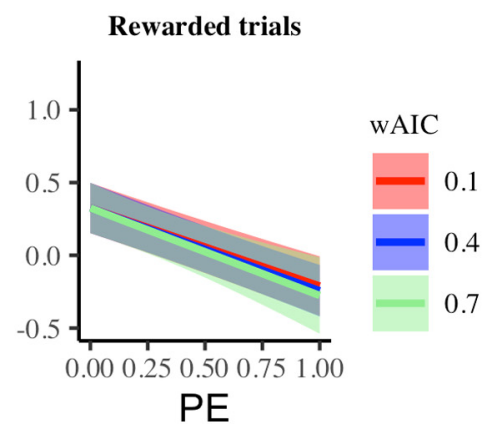

Figure 4. Result of linear regression between power and prediction error (PE) in all clusters. Lines indicate the trial-by-trial relation between the estimated PEs and the mean power extracted from the clusters in Figure 3. Shades represent $95 \%$ Cls. The model cluster $(\boldsymbol{A})$ aimed to predict empirical data from the theta cluster $(\boldsymbol{B})$. For exploratory purposes, also the relation between estimated PEs and power in the $\delta(\boldsymbol{C})$ and $\alpha(\boldsymbol{D})$ cluster.

However, the regression model that also included the three-way interaction between prediction error, reward, and wAIC fitted significantly better than the regression model with no interaction terms $\left(\chi^{2}(3, N=27)=9.27, R^{2}=0.028, p=0.026\right)$. Here, the main effect of prediction error was significant $\left(\chi^{2}(1, N=\right.$ $\left.27)=580, R^{2}=0.220, p<0.001, \beta=0.45\right)$. The interaction between prediction error and reward did not reach significance $\left(\chi^{2}(1, N=27)=3.49, R^{2}=0.017, p=0.062, \beta=-0.07\right)$. Also, the three-way interaction term did not reach significance $\left(\chi^{2}(2\right.$, $\left.N=27)=5.83, R^{2}=0.022, p=0.054\right)$. However, if the interaction was considered separately for rewarded trials $\left(R^{2}=\right.$ $0.020, \beta=0.61, p=0.018)$ and unrewarded trials $\left(R^{2}=0.015\right.$, $\beta=-0.50, p=0.033)$, both reached significance. As can be observed in Figure $4 C$, this meant that there was a positive linear relationship between power and prediction error for both positive and negative prediction error (Fig. 4C). For subjects with low wAIC, the slope in unrewarded trials was similar to the slope in rewarded trials, whereas for subjects with high wAIC, an inverse effect of the theta cluster was observed in which there was a flat slope in unrewarded trials but a steeper slope in rewarded trials.

In the $\alpha$ cluster, the regression model with the two-way interaction term showed a significantly better fit than the regression model without interaction $\left(\chi^{2}(1, N=27)=224, R^{2}=0.137\right.$, $p<0.001)$. When the three-way interaction was added, it did not lead to a significantly better regression model $\left(\chi^{2}(2, N=27)=\right.$ $\left.0.350, R^{2}=0.005, p=0.841\right)$. Here, a significant main effect of prediction error $\left(\chi^{2}(1, N=27)=142, R^{2}=0.109, p<0.001, \beta=\right.$ $0.85)$ and a significant interaction between prediction error and reward $\left(\chi^{2}(1, N=27)=226, R^{2}=0.137, p<0.001, \beta=-1.38\right)$ were observed. The three-way interaction between prediction error, reward, and wAIC was not significant $\left(\chi^{2}(2, N=27)=\right.$ $\left.0.360, R^{2}=0.005, p=0.833\right)$. As is shown in Figure $4 D$, power in the $\alpha$ cluster exhibited a positive linear relationship for negative prediction error, but a negative linear relationship with positive prediction error. These effects did not differ with respect to wAIC.

To explore the topology of these interaction effects described above, we conducted another cluster analysis. Here, we multiplied prediction error (scaled separately for positive and negative prediction errors) with reward ( -1 for unrewarded trials and 1 for rewarded trials) as a regressor for power. This resulted in a contrast value for the interaction between prediction error and reward for each electrode, time point, and frequency. These contrast values were then entered into the clustering algorithm. As expected, we observed a significant cluster in the $\alpha$ frequency (Fig. 5B), which was strongest on posterior electrodes (Fig. $5 C$ ). We also observed significant effects in the theta and $\delta$ frequency ranges (Fig. 5D). Although the interaction pattern for theta (Fig. $4 B$ ) and $\delta$ (Fig. 4C) are mirrored (and thus qualitatively different), they are represented by a similar contrast value because, in both theta and $\delta$ empirical patterns, the slope for positive prediction errors is larger than the slope for negative prediction errors. Because they are also topographically (partially) overlapping, they were clustered together by the algorithm, resulting in one cluster that was a mixture of the theta and $\delta$ effects on both time-frequency and topographical level.

In sum, in line with model predictions, we found a linear relationship between postfeedback theta power and negative prediction errors but not with positive prediction errors (interaction between reward and prediction error). Moreover, we found that this interaction effect was stronger for participants that fitted better with the bSync model. On top of model predictions, two other clusters could be distinguished in postfeedback power. Here, a $\delta$ cluster showed an almost exactly mirrored pattern 

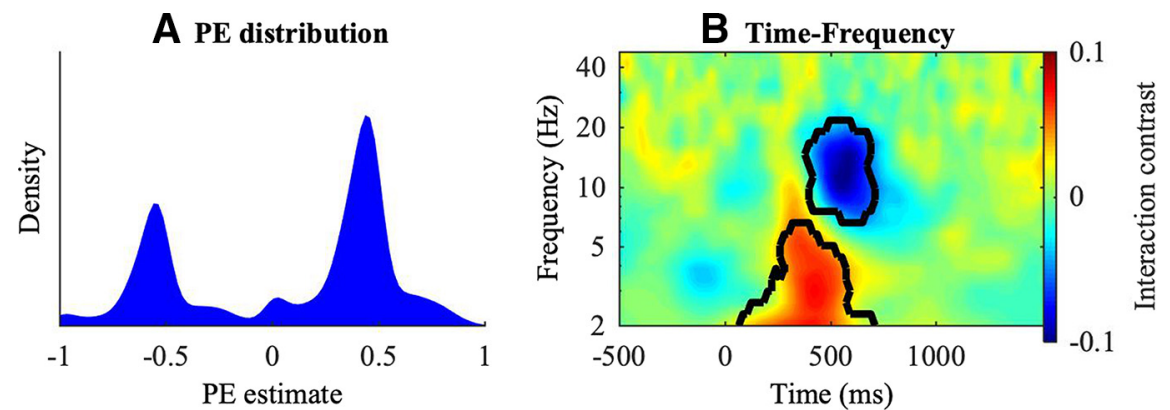

C Alpha cluster
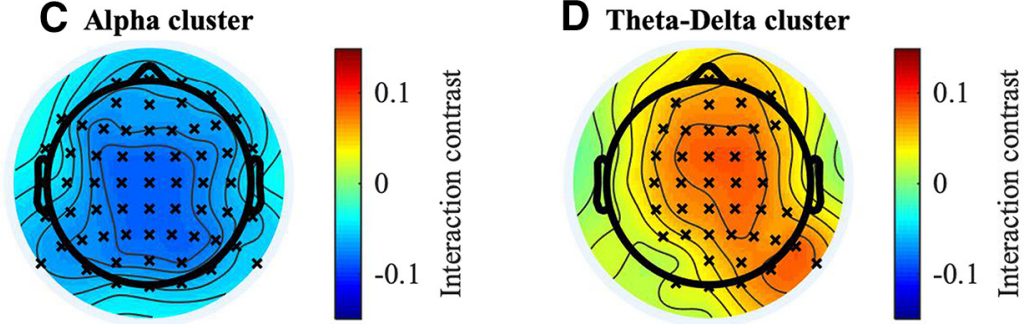

Figure 5. Interaction between prediction error and reward in power. $A$, Distribution of prediction error (PE) estimates. These prediction error estimates are not used for learning stimulus-action pairs, but for module learning in the Switch unit (Eq. 4). $\boldsymbol{B}$, Time-frequency results. Colors represent the contrast value of the interaction effect. Black contours indicate significant clusters. $\boldsymbol{C}$, Topography of the $\alpha$ interaction cluster. $\boldsymbol{D}$, Topography of the theta- $\delta$ interaction cluster. Crosses represent channels where the contrast reached significance.

relative to the theta cluster. An $\alpha$ cluster showed an inversed $\mathrm{U}$-shaped pattern with respect to prediction errors.

\section{Rule switch locking}

For the second model-driven EEG hypothesis, power from the theta, $\alpha$, and $\delta$ clusters was extracted in trials within a 31-trial window from the rule switch ( -15 to 15$)$. In all clusters, one trial significantly deviated from baseline power. In the theta cluster (Fig. 6A), only the rule switch (0; i.e., all trials exactly at rule switch) was significant above baseline $\left(t_{(26)}=4.378, R^{2}=0.424\right.$, CI 99.84 [-2.059, 0.256], baseline $=-2.340)$. Linear regression of the data time course (across 31 trials) on the Sync model time course showed a significant effect $\left(F_{(1,835)}=20.51, p<0.001\right.$, $R_{\text {adj }}^{2}=0.023, \beta=0.31$ ). In the $\delta$ cluster (Fig. $6 B$ ), only the rule switch $(0)$ was significantly below baseline $\left(t_{(26)}=-3.890, R^{2}=\right.$ 0.368, CI 99.84 [-2.450, -1.265], baseline $=-1.201)$. Linear regression of the data time course on the Sync model time course revealed a significant correlation $\left(F_{(1,835)}=7.36, p=0.007, R_{\text {adj }}^{2}=\right.$ $0.008, \beta=-0.18$ ). For the $\alpha$ cluster (Fig. $6 C$ ), again one trial was significantly below baseline $\left(t_{(26)}=-2.698, R^{2}=0.219\right.$, CI 99.84 $[-6.275,-3.603]$, baseline $=-3.584)$. Notably, this was the point right after the rule switch (1; i.e., all trials right after the rule switch). Moreover, when data were locked to the moment where subjects indicated the rule switch (Fig. $6 D$ ), alpha power reaches a minimum at this exact moment $\left(t_{(26)}=-3.703, R^{2}=0.345, \mathrm{CI}\right.$ $99.84[-7.675,-3.686]$, baseline $=-3.584)$. Also in the $\alpha$ cluster, the linear regression of the power on the Sync model pattern reached significance with a negative slope $\left(F_{(1,835)}=32.72\right.$, $\left.p<0.001, R^{2}=0.037, \beta=-0.65\right)$.

Power at the peak trials (trials at point 0 for theta and $\delta$, trials at point 1 for $\alpha$ ) was extracted and added to a linear regression with wAIC as predictor. This revealed no significant effects for the theta $\left(F_{(1,25)}=0.004, p=0.948, R^{2}=-0.040, \beta=-0.10\right)$ or $\delta$ cluster $\left(F_{(1,25)}=0.680, p=0.417, R^{2}=-0.012, \beta=0.66\right)$. However, the effect of wAIC did reach significance in the $\alpha$ cluster $\left(F_{(1,25)}=7.22, p=0.013, R^{2}=0.193, \beta=4.17\right)$. Figure 7 sheds light on how activity in the $\alpha$ cluster differed depending on wAIC. For illustrative purposes, subjects were divided in three groups of low, middle, and high wAIC. For each group, the data pattern of alpha activity was plotted, once locked to the real rule switch (Fig. $7 A$ ) and once locked to the indication of a rule switch (Fig. $7 B$ ). Here, it is observed that the $\alpha$ pattern is mainly driven by subjects with a low wAIC (i.e., bad fit) for the bSync model.

In sum, simulated theta power significantly predicted empirical theta power. Here, theta power peaked at the moment of a rule switch. Just like in the first model-driven EEG hypothesis, power from the empirical $\delta$ cluster showed the mirrored pattern compared with theta. Remarkably, $\alpha$ cluster showed a dip in power, not with respect to the actual rule switch but with respect to the subjectively indicated rule switch.

\section{Midfrontal-posterior phase-coupling analyses}

We next turn to our third modeldriven EEG analysis concerning an increase of phase-coupling between midfrontal and posterior electrodes after negative feedback. As previously described, in the Sync model, this coupling is induced by bursts that are sent from pMFC to posterior areas in the Mapping unit. Since pMFC power is stronger after negative feedback, also the number of bursts and the amount of phase-coupling are increased. To investigate this, we looked at phase-coupling between a midfrontal electrode (FCz) and all lateral electrodes.

Here, nonparametric cluster analyses on the phase-locking data (Fig. 8) revealed six significant clusters that were selective for feedback (for details, see Materials and Methods). These clusters were located in the theta (4; Fig. $8 A-C)$ or $\delta(2$; Fig. $8 A, B, D)$ frequency band. In the theta frequency band, two clusters were located at temporal electrodes; two other clusters were located on more lateral/anterior frontal electrodes. In the $\delta$ frequency band, both clusters were located on posterior electrodes. In line with the results of Sync model simulations (Fig. $8 E$ ), the theta clusters showed an increase in phase-locking after negative feedback. This was the case for both the ipsilateral and contralateral electrodes. The $\delta$ clusters show the inverse pattern of the theta cluster. Here, phase-locking was stronger after positive feedback than after negative feedback in both the ipsilateral and contralateral cluster. As in the power analyses, we also explored whether the phase-locking contrast in each cluster correlated with the subjects' wAIC for the bSync model. None of these correlations reached significance.

In sum, as predicted by the Sync model, we found stronger phase-coupling in the theta frequency between midfrontal and more posterior electrodes after negative feedback than after positive feedback. Additionally, we found an increase in phase-coupling between midfrontal and lateral frontal electrodes, also in the theta frequency range. Similar to our power analyses, we found an inverse effect in the $\delta$ frequency compared with the theta frequency.

\section{Discussion}

The current study aimed to gain insight in the neural mechanisms that allow humans to flexibly adapt to rule changes in the environment. Twenty-seven healthy human subjects were tested 
on a probabilistic reversal learning task while measuring EEG. Behaviorally, three models of increasing hierarchical complexity were compared. A first, RW model, updated the value of stimulusaction mappings on a trial-by-trial basis with a fixed learning rate. In a second, ALR model, this approach was extended with an adaptable learning rate, allowing the ALR model to flexibly adapt to rule switches (fast learning) but to also be robust to noise evoked by probabilistic feedback (slow learning). The third, Sync model implemented modularity to retain task-specific mappings. It uses hierarchical learning to determine when to switch between rule modules. No evidence was found for the ALR model, while for some subjects the RW model fit best, and for others the Sync model.

Simulations of the Sync model allowed formulation and testing of three model-driven EEG hypotheses. The first hypothesis concerns midfrontal theta and prediction errors. In the Sync model, prediction errors are used to evaluate how much control is needed. The level of control is represented by theta power in the pMFC. Since only negative prediction errors inform about rule switches, the Sync model increased control after negative prediction errors but not after positive prediction errors. Thus, although most previous work (Cavanagh et al., 2009, 2010; Ergo et al., 2019) described a U-shape relationship between prediction error and theta power, we hypothesized and observed a selectivity for negative prediction errors (see also Janssen et al., 2016). A linear relationship between prediction error and power in the theta cluster was observed for unrewarded trials (negative prediction error) but not for rewarded trials (positive prediction error). This effect was stronger for subjects with a better Sync model fit. Based on our theoretically driven hypothesis, we did not extract prediction errors from stimulus-action learning but from learning in the Switch unit. Future research should investigate how midfrontal theta is influenced by different types of prediction errors.

Since prediction errors are strongest at the rule switch, a second model-driven hypothesis stated that theta power peaks at rule switches. Again, this hypothesis was empirically supported. Moreover, simulated power significantly predicted power in the empirical theta cluster. Consistent with earlier work (Sauseng et al., 2006; Cunillera et al., 2012), theta power increased and alpha power decreased at rule switches. How this theta increase relates to the $\alpha$ decrease, and to the individual differences that we observed, deserves future research.

Current work provides a mechanistic explanation how increases in theta power after prediction errors implement new task rules by synchronizing modules. This resulted in a third model-driven hypothesis. Here, the Sync model uniquely predicted that phase connectivity would increase after negative feedback. We found six significant clusters. Four of them were in theta frequency range and showed the predicted pattern. Two of these clusters were located on posterior-temporal electrodes, roughly in line with our prediction of motor and visual areas. The remaining four clusters were consistent with previous work (Cavanagh et al., 2010) showing a feedback-locked, prediction error-induced increase of theta phase-coupling between midfrontal and lateral frontal sites, and a $\delta$ coupling decrease between midfrontal and posterior cortical sites.

Several hypotheses remain to be tested. First, as mentioned in Materials and Methods, previous modeling work (Verbeke and Verguts, 2019) used $\gamma$ frequency in the Mapping unit
B Delta cluster

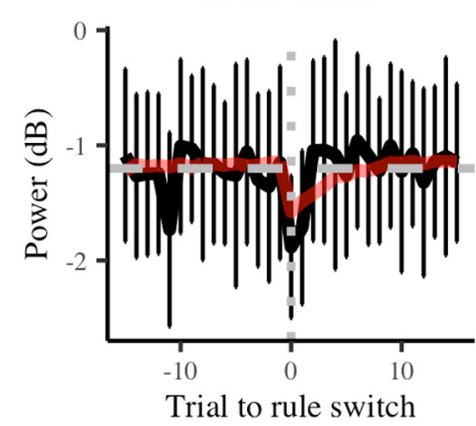

D Alpha to indicated switch

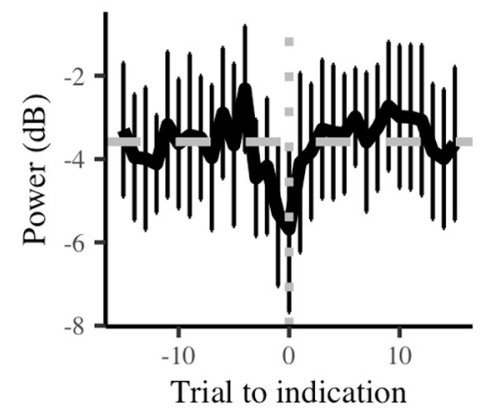

Trial to indication
Theta cluster

Alpha cluster

Trial to rule switch . $9.84 \%$

Figure 6. Power locked to rule switch. Black lines indicate the mean power. Error bars indicate the $99.84 \%$
$\mathrm{Cl}$ (Bonferroni correction). Horizontal gray dashed line indicates baseline power. Vertical gray dotted line indicates the moment of the rule switch. Red line visualizes the result of linear regression between the Sync model and human data. $\boldsymbol{A}$ - $\boldsymbol{C}$, Data locked to the moment of the actual rule switch. $\boldsymbol{D}$, Data of the $\alpha$ cluster locked to . $\begin{array}{llll} & & & \\ \text { wAIC } & 0.1 & 0.4=0.7\end{array}$
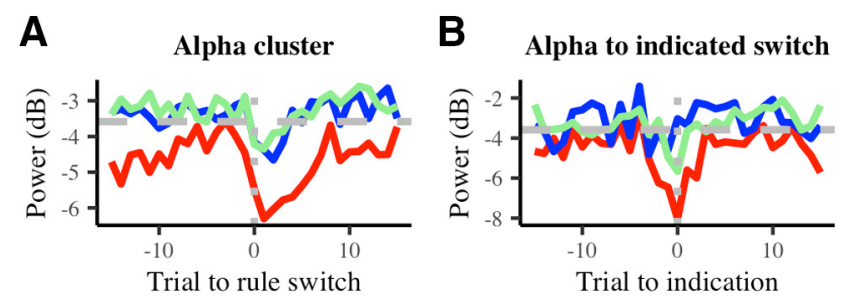

Figure 7. Power locked to rule switch for different wAIC. Data patterns are shown for different wAIC values (colored lines). Horizontal gray dashed line indicates the baseline power over all subjects. Vertical gray dotted line indicates the moment of the rule switch $(\boldsymbol{A})$ or indication of rule switch $(\boldsymbol{B})$.

instead of theta frequency. This frequency was currently changed because empirical work demonstrated within-frequency (theta-theta) coupling (Cavanagh et al., 2009; Clouter et al., 2017) during cognitive tasks, in addition to cross-frequency coupling. We thus also studied within-frequency coupling empirically. Nevertheless, future work, using MEG or more invasive measurements, should also study the role of cross-frequency (theta- $\gamma$ ) coupling. Second, the limited spatial resolution of EEG did not allow testing whether task rules are implemented by synchronizing task-relevant modules.

Several model extensions can be made. For instance, while for the current reversal learning task it was sufficient to use prediction error to determine when to make a binary switch, a more sophisticated approach might apply in everyday life, where contextual cues allow navigating a vast map of tasks and rules. One way to address this issue is by adding secondlevel contextual features, which allow the LFC to (learn to) 

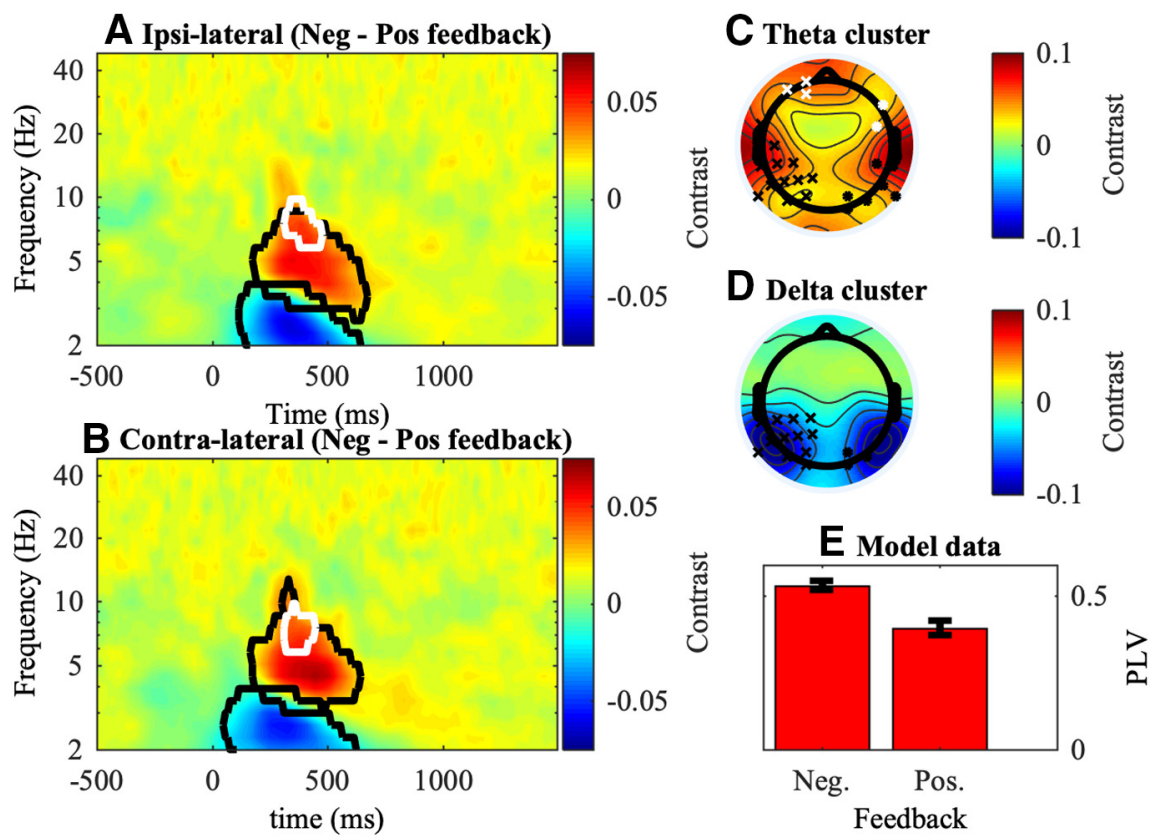

Figure 8. Phase-locking with respect to FCZ. A, B, Time-frequency plots of contrast (Negative - Positive feedback). Black or white contour line indicates significant clusters. Black line indicates posterior clusters in $\boldsymbol{C}$ and $\boldsymbol{D}$ (black crosses). White line indicates the frontal clusters in $\boldsymbol{C}$ (white crosses). All plots represent phase-locking with respect to the FGz electrode. $\boldsymbol{A}$, Contrast of iPLV averaged over all ipsilateral electrodes. $\boldsymbol{B}$, Contrast of iPLV averaged over all contralateral electrodes. $\boldsymbol{C}, \boldsymbol{D}$, Topographical plots of clusters. Data were averaged over all time points and frequencies that were included in the respective contours of $\boldsymbol{A}$ and $\boldsymbol{B}$. Crosses or dots represent channels where the contrast reached significance. Left channels (crosses) present ipsilateral electrodes. Right channels (dots) present contralateral electrodes. Again, white distinguishes the frontal clusters from the temporal clusters. $E$, Predicted phase-coupling in the model for the 250-500 ms postfeedback period.

infer which of multiple task modules should be synchronized. Additionally, scalability of the Sync model is currently limited by how modularity was implemented in the Mapping unit. Here, none of the Rule 1 mappings are shared with Rule 2. Such a strict division of task mappings is optimal when mappings are orthogonal. However, when some mappings can be generalized between tasks, the current approach does not allow knowledge transfer across contexts. As addressed previously (Gershman et al., 2010; Collins and Frank, 2013), a more sustainable way is to construct modules of mappings that are shared between tasks. Instead of learning each new task from scratch, this approach allows transferring partial knowledge between tasks. Future work should explore whether these more complex hierarchical learning algorithms can be integrated in the Sync model.

Recent work emphasized that reinforcement learning can operate not only over observed states, but also over belief states that an agent may infer (Wilson et al., 2014; Gershman and Uchida, 2019). In the Sync model, there were no (contextual) cues. Therefore, the Sync model could rely exclusively on prediction errors to estimate the belief state (task rule) of the environment. When contextual features are added, a future version of the Sync model may estimate belief states in a more efficient manner. Also, the Sync model uses two types of prediction error: one to adjust lower-level mappings and another to determine the (higher-level) task rule state. Instead, nonhierarchical models (e.g., RW, ALR) use prediction errors only to adjust lower-level mappings.

Building on suggestions of previous work (Piray et al., 2019), the current study illustrated how individual differences in model fit can be leveraged to address cognitive questions. Three groups were distinguished: one group aligned with the RW model, a second group aligned with the Sync model, and a third group in which the RW and Sync model could not be empirically distinguished. Although the differences between groups were nonsignificant when averaging over several trials (e.g., learning curve), more fine-grained measures (e.g., wAIC, trialby-trial power) revealed important individual differences. Interestingly, subjects with lower accuracy fitted better with the Sync model. This is consistent with previous work (Verbeke and Verguts, 2019), which illustrated that modularity as used by the Sync model is only beneficial if the learning problem is sufficiently complex. Furthermore, despite previous work showing a good behavioral fit of the ALR model (Bai et al., 2014), the fit of the ALR model in the current study was consistently low. In contrast to previous studies, the current task applied more frequent task rule switches without long stable trial blocks, favoring constant high learning rates. Thus, future work should investigate whether subjects use the RW, ALR, or Sync framework depending on the structure and complexity of the task.

The Sync model implements modularity via neural oscillations between task-relevant areas. This concords with a role of neural oscillations for a wide variety of cognitive functions, including visual attention (Gray and Singer, 1989; Jensen et al., 2012), working memory (Lisman and Idiart, 1995; Hsieh et al., 2011), cognitive control (Cavanagh and Frank, 2014), and declarative learning (Ergo et al., 2020). According to the BBS hypothesis (Fries, 2015), these cognitive functions require binding of several stimuli or features. Current work described how oscillations, and more specifically synchronization, might be relevant in hierarchical rule learning.

On anatomic-functional level, we built on suggestions from previous work that pMFC cooperates with LFC to exert hierarchical control over lower-level motor processes (Koechlin et al., 2003; Alexander and Brown, 2015). In the Sync model, LFC signals which rule modules should be synchronized. Consistently, previous theories describe LFC as containing task demands (Botvinick et al., 2001), and empirical work found strong communication between LFC and pMFC in cognitive tasks (MacDonald et al., 2000; Kondo et al., 2004; Cavanagh et al., 2010). Also in line with previous data (Boorman et al., 2009; Wilson et al., 2014; Holroyd and McClure, 2015), the model aMFC keeps track of the relevant task rule. Additionally, consistent with fMRI work (Aben et al., 2020), the current study found increased coupling between midfrontal cortex and task-related areas when more control was needed (negative feedback). While this fMRI work showed anatomically detailed networks of connectivity, the current study described how this connectivity may work at algorithmic level.

In conclusion, we have demonstrated how the brain might use synchronization to bind task-relevant areas for efficient rule switching. To achieve this, we used EEG, computational modeling, individual differences, and behavioral analysis. We believe that this approach might reveal how more complicated tasks can be implemented via synchronization as well.

\section{References}

Aben B, Calderon CB, Van den Bussche E, Verguts T (2020) Cognitive effort modulates connectivity between dorsal anterior cingulate cortex and task-relevant cortical areas. J Neurosci 40:3838-3848. 
Alexander WH, Brown JW (2015) Hierarchical error representation: a computational model of anterior cingulate and dorsolateral prefrontal cortex. Neural Comput 27:2354-2410.

Bai Y, Katahira K, Ohira H (2014) Dual learning processes underlying human decision-making in reversal learning tasks: functional significance and evidence from the model fit to human behavior. Front Psychol 5:871-878.

Behrens TE, Woolrich MW, Walton ME, Rushworth MF (2007) Learning the value of information in an uncertain world. Nat Neurosci 10:1214-1221.

Boorman ED, Behrens TE, Woolrich MW, Rushworth MF (2009) How green is the grass on the other side? Frontopolar cortex and the evidence in favor of alternative courses of action. Neuron 62:733-743.

Botvinick MM, Braver TS, Barch DM, Carter CS, Cohen JD (2001) Conflict monitoring and cognitive control. Psychol Rev 108:624-652.

Bruña R, Maestú F, Pereda E (2018) Phase locking value revisited: teaching new tricks to an old dog. J Neural Eng 15:056011.

Cavanagh JF, Frank MJ (2014) Frontal theta as a mechanism for cognitive control. Trends Cogn Sci 18:414-421.

Cavanagh JF, Cohen MX, Allen JJ (2009) Prelude to and resolution of an error: EEG phase synchrony reveals cognitive control dynamics during action monitoring. J Neurosci 29:98-105.

Cavanagh JF, Frank MJ, Klein TJ, Allen JJ (2010) Frontal theta links prediction errors to behavioral adaptation in reinforcement learning. Neuroimage 49:3198-3209.

Clouter A, Shapiro KL, Hanslmayr S (2017) Theta phase synchronization is the glue that binds human associative memory. Curr Biol 27:3143-3146.

Cohen JD, Dunbar K, McClelland JL (1990) On the control of automatic processes: a parallel distributed processing account of the Stroop effect. Psychol Rev 97:332-361.

Cohen MX (2014) Analyzing neural time series data: theory and practice. Cambridge, MA: Massachusetts Institute of Technology.

Collins AG, Frank MJ (2013) Cognitive control over learning: creating, clustering, and generalizing task-set structure. Psychol Rev 120:190-229.

Cools R, Clark L, Owen AM, Robbins TW (2002) Defining the neural mechanisms of probabilistic reversal learning using event-related functional magnetic resonance imaging. J Neurosci 22:4563-4567.

Cunillera T, Fuentemilla L, Periañez J, Marco-Pallarès J, Krämer UM, Càmara E, Münte TF, Rodríguez-Fornells A (2012) Brain oscillatory activity associated with task switching and feedback processing. Cogn Affect Behav Neurosci 12:16-33.

Delorme A, Makeig S (2004) EEGLAB: an open source toolbox for analysis of single-trial EEG dynamics including independent component analysis. J Neurosci Methods 134:9-21.

Ergo K, De Loof E, Janssens C, Verguts T (2019) Oscillatory signatures of reward prediction errors in declarative learning. Neuroimage 186:137-145.

Ergo K, De Loof E, Verguts T (2020) Reward prediction error and declarative memory. Trends Cogn Sci 24:388-397.

French RM (1999) Catastrophic forgetting in connectionist networks. Trends Cogn Sci 3:128-135.

Fries P (2005) A mechanism for cognitive dynamics: neuronal communication through neuronal coherence. Trends Cogn Sci 9:474-480.

Fries P (2015) Rhythms for cognition: communication through coherence. Neuron 88:220-235.

Gershman SJ, Blei DM, Niv Y (2010) Context, learning, and extinction. Psychol Rev 117:197-209.

Gershman SJ, Uchida N (2019) Believing in dopamine. Nat Rev Neurosci 20:703-714

Gray CM, Singer W (1989) Stimulus-specific neuronal oscillations in orientation columns of cat visual cortex. Proc Natl Acad Sci USA 86:1698-1702.

Holroyd CB (2016) The waste disposal problem of effortful control. In: Motivation and cognitive control (Braver TS, ed), pp 235-260. Hove, UK: Psychology.

Holroyd CB, McClure SM (2015) Hierarchical control over effortful behavior by rodent medial frontal cortex: a computational model. Psychol Rev 122:54-83.

Hsieh LT, Ekstrom AD, Ranganath C (2011) Neural oscillations associated with item and temporal order maintenance in working memory. J Neurosci 31:10803-10810.

Izquierdo A, Brigman JL, Radke AK, Rudebeck PH, Holmes A (2017) The neural basis of reversal learning: an updated perspective. Neuroscience $345: 12-26$
Janssen DJ, Poljac E, Bekkering H (2016) Binary sensitivity of theta activity for gain and loss when monitoring parametric prediction errors. Soc Cogn Affect Neurosci 11:1280-1289.

Jasper H (1958) The ten twenty electrode system of the international federation. Electroencephalogr Clin Neurophysiol Suppl 52:3-6.

Jensen O, Bonnefond M, VanRullen R (2012) An oscillatory mechanism for prioritizing salient unattended stimuli. Trends Cogn Sci 16:200-205.

Koechlin E, Ody C, Kouneiher F (2003) The architecture of cognitive control in the human prefrontal cortex. Science 302:1181-1185.

Kondo H, Osaka N, Osaka M (2004) Cooperation of the anterior cingulate cortex and dorsolateral prefrontal cortex for attention shifting. Neuroimage 23:670-679.

Lachaux JP, Rodriguez E, Martinerie J, Varela FJ (1999) Measuring phase synchrony in brain signals. Hum Brain Mapp 8:194-208.

Lisman JE, Idiart MA (1995) Storage of $7 \pm 2$ short-term memories in oscillatory subcycles. Science 267:1512-1515.

MacDonald AW, Cohen JD, Stenger AV, Carter CS (2000) Dissociating the role of the dorsolateral prefrontal and anterior cingulate cortex in cognitive control. Science 288:1835-1838.

Makoto M (2018) Makoto's preprocessing pipeline. Available at https://sccn. ucsd.edu/wiki/Makoto\%27s_preprocessing_pipeline.

Maris E, Oostenveld R (2007) Nonparametric statistical testing of EEG- and MEG-data. J Neurosci Methods 164:177-190.

Nolte G, Bai O, Wheaton L, Mari Z, Vorbach S, Hallett M (2004) Identifying true brain interaction from EEG data using the imaginary part of coherency. Clin Neurophysiol 115:2292-2307.

Peirce J, Gray JR, Simpson S, MacAskill M, Höchenberger R, Sogo H, Kastman E, Lindeløv JK (2019) PsychoPy2: experiments in behavior made easy. Behav Res Methods 51:195-203.

Piray P, Dezfouli A, Heskes T, Frank M, Daw N (2019) Hierarchical Bayesian inference for concurrent model fitting and comparison for group studies. PLoS Comput Biol 15:e1007043.

R Core Team (2017) R: a language and environment for statistical computing. Vienna: R Core Team.

Rescorla RA, Wagner AR (1972) A theory of Pavlovian conditioning: variations in the effectiveness of reinforcement and nonreinforcement. In: Classical conditioning: II. Current research and theory, pp 64-99. New York: Appleton-Century-Crofts.

Saez A, Rigotti M, Ostojic S, Fusi S, Salzman CD (2015) Abstract context representations in primate amygdala and prefrontal cortex. Neuron 87:869-881.

Sauseng P, Klimesch W, Freunberger R, Pecherstorfer T, Hanslmayr S, Doppelmayr M (2006) Relevance of EEG alpha and theta oscillations during task switching. Exp Brain Res 170:295-301.

Silvetti M, Seurinck R, Verguts T (2011) Value and prediction error in medial frontal cortex: integrating the single-unit and systems levels of analysis. Front Hum Neurosci 5:75.

Silvetti M, Vassena E, Abrahamse E, Verguts T (2018) Dorsal anterior cingulate-brainstem ensemble as a reinforcement meta-learner. PLoS Comput Biol 14:e1006370.

Springer M, Paulsson J (2006) Harmonies from noise. Nature 439:27-29.

Verbeke P, Verguts T (2019) Learning to synchronize: how biological agents can couple neural task modules for dealing with the stability-plasticity dilemma. PLoS Comput Biol 15:e1006604.

Verguts T (2017) Binding by random bursts: a computational model of cognitive control. J Cogn Neurosci 29:1103-1118.

Widrow B, Hoff MM (1960) Adaptive switching circuits. IRE WESCON Convention Record 4:96-104.

Wilson RC, Takahashi YK, Schoenbaum G, Niv Y (2014) Orbitofrontal cortex as a cognitive map of task space. Neuron 81:267-279.

Womelsdorf T, Schoffelen J, Oostenveld R, Singer W, Desimone R, Engel AK, Fries $P$ (2007) Modulation of neuronal interactions through neuronal synchronization. Science 316:1609-1612.

Womelsdorf T, Johnston K, Vinck M, Everling S (2010) Theta-activity in anterior cingulate cortex predicts task rules and their adjustments following errors. Proc Natl Acad Sci USA 107:5248-5253.

Zhou T, Chen L, Aihara K (2005) Molecular communication through stochastic synchronization induced by extracellular fluctuations. Phys Rev Lett 95:178103. 